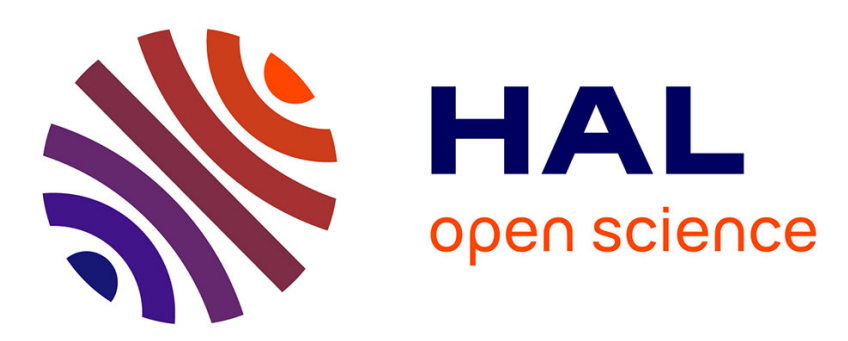

\title{
Productivity analysis and non-linear gain scheduling approach for multi-species bioprocesses with product inhibition
}

Piotr Skupin, Alain Rapaport

\section{To cite this version:}

Piotr Skupin, Alain Rapaport. Productivity analysis and non-linear gain scheduling approach for multi-species bioprocesses with product inhibition. IEEE Conference on Decision and Control CDC2019, Dec 2019, Nice, France. pp.7-12, 10.1109/CDC40024.2019.9028869 hal-02434291

\section{HAL Id: hal-02434291 \\ https://hal.science/hal-02434291}

Submitted on 9 Jan 2020

HAL is a multi-disciplinary open access archive for the deposit and dissemination of scientific research documents, whether they are published or not. The documents may come from teaching and research institutions in France or abroad, or from public or private research centers.
L'archive ouverte pluridisciplinaire HAL, est destinée au dépôt et à la diffusion de documents scientifiques de niveau recherche, publiés ou non, émanant des établissements d'enseignement et de recherche français ou étrangers, des laboratoires publics ou privés. 


\title{
Productivity analysis and non-linear gain scheduling approach for multi-species bioprocesses with product inhibition
}

\author{
Piotr Skupin and Alain Rapaport
}

\begin{abstract}
Bioprocesses with product inhibition are known to allow species coexistence. In this work, we first study the productivity of the different possible equilibria, depending on the operating conditions, and show that single species offers the best performances. Then, we propose a control strategy to stabilize the dynamics about the desired equilibria, in presence of instability. Based on output feedback linearization, we propose a family of controllers and a gain-scheduling approach to adapt the controller. Finally, we illustrate our approach on numerical simulations, showing that the attraction basin of the closed-loop system is improved by considering the gainscheduling approach.
\end{abstract}

\section{INTRODUCTION}

The chemostat is an experimental apparatus for continuous cultivation of microorganisms that ensures constant environmental conditions. Therefore, the chemostat is an important tool for studying relationships between several competing species and is also widely used in biotechnological industry to produce high-value products at constant inlet flow rates. It is well-known that in the classical chemostat model with two competing species for a single nutrient, the coexistence is not possible (under specific growth rates which are only resource dependent such as the Monod law, and constant yield coefficient; see for instance [1], [2]). Many studies have shown that the coexistence of two competing species is possible in the presence of internal or external inhibitor in the system [3], [4], [5]. For instance, in the paper by De Freitas and Fredrickson (1978) [6], two species were competing for a single growth limiting substrate in the presence of internal inhibitor. The inhibitor was produced by both species and this case is typical for ethanol fermentation processes, where different strains of yeast produce ethanol that inhibits their growth. Since the product yield or sensitivity to inhibitory product can be different for both species, one can observe the following cases: only one of the species wins the competition (depending on the operating conditions: dilution rate and input concentration), both species can coexist or the coexistence is not possible, or only one of the species wins the competition (depending on the initial species concentrations). In the last case, we deal with multiplicity of steady-states and the desired product concentration may significantly differ, depending on which species wins the competition.

In the continuous stirred bioreactor, a typical control task is the maximization of its productivity, which is defined as the amount of product produced per unit volume per

P. Skupin is with Silesian University of Technology, Faculty of Automatic Control, Electronics and Computer Science, Gliwice, Poland piotr.skupin@polsl.pl, A. Rapaport is with MISTEA, Univ Montpellier, INRA, France alain.rapaporteinra.fr unit time. The control task can be achieved by setting the dilution rate in a sub-optimal region [7]. This is due to a fact that the optimal dilution rate is usually located near a critical dilution rate, above which the washout occurs. To avoid a risk of washing the biomass out of the reactor vessel, a closed-loop controller can be designed to stabilize the productivity directly or indirectly, by stabilizing only the product or biomass concentrations. Due to the nonlinear nature of bioprocesses, the classical PI controllers may be inefficient for this task, especially in the presence of larger magnitude disturbances [8]. Therefore, more effective control strategies have been developed for controlling key process variables in bioprocesses and these include: nonlinear feedback controller [9], model predictive control [10], [11] or input-output linearizing controllers [7], [12], [13], [14].

The linearizing control algorithms can be very efficient, but the resulting closed-loop system may not be controllable at every steady-state and the manipulated variable may take unphysical values [15]. In fact, the manipulated variable is always constrained and the control signal saturations should be taken into account in the controller design [14], [16], [17]. Keeping these facts in mind, our goal is to design a feedback linearizing controller for stabilization of the product concentration (thus, the productivity) in continuous culture with two competing species for a single nutrient. We consider a chemostat model from [6], where both competing species secrete a desired product and the product inhibits their growth. In comparison to the results presented in [6], we consider more than one coexistence equilibrium point, show global stability of the chemostat system and perform the analysis of productivity, leading to a stabilization problem. Since the product yield is different for each of the species, it is shown that the best productivity can only be obtained for a single species case. The proposed controller ensures that the desired productivity can be achieved, also in the case of multiplicity of steady-states. Then, we propose a gainscheduling approach to increase the convergence rate to a desired set point product concentration. The effectiveness of the controller is presented based on numerical simulations, showing that the attraction basin of the closed-loop system is improved by the gain-scheduling approach. The rest of the paper is organized as follows. In Section II the productivity of the open-loop system is analyzed. Section III presents the idea of the proposed control algorithm and Section IV presents simulation results. Finally, Section V concludes the paper. 


\section{Productivity ANALYSIS IN OPEN-LOOP}

We consider the chemostat model with two species, of density $X_{1}, X_{2}$ competing for a single resource of concentration $S$ in the chemostat, subject of dilution rate $D>0$ which constantly brings the resource with concentration $S_{i n}$ :

$$
\begin{aligned}
\frac{d S}{d t} & =-\frac{1}{Y_{x 1}} \mu_{1}(S, P) X_{1}-\frac{1}{Y_{x 2}} \mu_{2}(S, P) X_{2}+D\left(S_{\text {in }}-S\right) \\
\frac{d X_{1}}{d t} & =\mu_{1}(S, P) X_{1}-D X_{1} \\
\frac{d X_{2}}{d t} & =\mu_{2}(S, P) X_{2}-D X_{2} \\
\frac{d P}{d t} & =Y_{p 1} \mu_{1}(S, P) X_{1}+Y_{p 2} \mu_{2}(S, P) X_{2}-D P
\end{aligned}
$$

Here, the species consume the substrate $S$ with conversion factor $Y_{x 1}, Y_{x 2}$ and produce $P$ with efficiency $Y_{p 1}, Y_{p 2}$. The particularity of this model is that the growth kinetics $\mu_{1}, \mu_{2}$ are inhibited by the product $P$, taking the expressions

$$
\mu_{1}(S, P)=\frac{\mu_{m 1} S}{K_{s 1}+S} \frac{K_{p 1}}{K_{p 1}+P}, \mu_{2}(S, P)=\frac{\mu_{m 2} S}{K_{s 2}+S} \frac{K_{p 2}}{K_{p 2}+P}
$$

To analyze the equilibria, one can follow the graphical method introduced in [6]. From equations (2), (3), one can see that the presence of species $X_{j}(j=1,2)$ at steady state implies that the pair $(S, P)$ at equilibrium belongs to the curve

$$
\mathscr{C}_{i}:=\left\{(S, P) \in \mathbb{R}_{+}^{2} \text { s.t. } \mu_{i}(S, P)=D\right\}
$$

in the $(S, P)$ plane. Moreover, when a species is not present at steady state, equations (1) and (4) imply that the pair $(S, P)$ belongs to the line

$$
\mathscr{L}_{i}:=\left\{(S, P) \in \mathbb{R}_{+}^{2} \text { s.t. } P=Y_{x i} Y_{p i}\left(S_{i n}-S\right)\right\}
$$

where $i$ denotes the label of the other species. Then, again from equations (1) and (4), coexistence is possible only when $P-Y_{x j} Y_{p j}\left(S_{i n}-S\right)$ or $-\left(P-Y_{x i} Y_{p i}\left(S_{i n}-S\right)\right)$ and $Y_{x i} Y_{p i}-$ $Y_{x j} Y_{p j}$ with $j \neq i$ have the same signs at steady state. Therefore, one can conclude about the existence of equilibra in a graphical way, as follows

- equilibria $E_{\star}$ with both species corresponds to intersections of $\mathscr{C}_{1}$ and $\mathscr{C}_{2}$ that lie in between $\mathscr{L}_{1}$ and $\mathscr{L}_{2}$,

- equilibrium $E_{i}$ with species $i$ alone corresponds to intersection of $\mathscr{C}_{i}$ and $\mathscr{L}_{i}$,

- the washout equilibrium $E_{0}$ always exists as intersection of $\mathscr{L}_{1}$ and $\mathscr{L}_{2}$, that is at $\left(S_{i n}, 0\right)$.

Notice that from the expressions (5), the equation $\mu_{i}(S, P)=$ $D$ amounts to have $P$ as a rational function of $S$ with degrees of numerator and denominator both equal to one. Then, the coexistence condition $\mu_{1}(S, P)=\mu_{2}(S, P)$ imposes $S$ to be solution of $a S^{2}+b S+c=0$, where the coefficients can be easily determined as

$$
\begin{aligned}
& a=\mu_{m 1} K_{p 1}-\mu_{m 2} K_{p 2}+D\left(K_{p 2}-K_{p 1}\right) \\
& b=\mu_{m 1} K_{s 2} K_{p 1}-\mu_{m 2} K_{s 1} K_{p 2}+D\left(K_{p 2}-K_{p 1}\right)\left(K_{s 1}+K_{s 2}\right) \\
& c=D\left(K_{p 2}-K_{p 1}\right) K_{s 1} K_{s 2}
\end{aligned}
$$
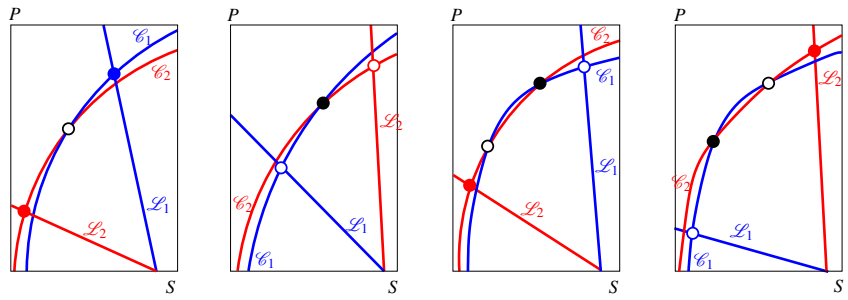

Fig. 1. The four different possibilities when coexistence equilibria exist (stable equilibrium with filled disk, unstable with empty disk)

leading to the possibility of having one or two coexistence equilibria. In [6], the possibility to have more than one coexistence equilibrium was mentioned but not studied.

In addition, the local stability of the equilibria can also be deduced graphically (see Fig. 1). One can notice that being above, resp. below $\mathscr{C}_{i}$ in the $(S, P)$ plane implies that $X_{i}$ is decreasing, resp. increasing, and then deduce the following stability rules:

- an equilibrium $E_{i}$ is stable if the intersection $\mathscr{C}_{i} \cap \mathscr{L}_{i}$ is above $\mathscr{C}_{j}$ with $j \neq i$.

- if there exists an unique equilibrium $E_{\star}$, it is stable exactly when $E_{1}$ and $E_{2}$ are unstable, and unstable when $E_{1}$ are $E_{2}$ are stable,

- when there exist two equilibria, one is stable and the other one unstable. Moreover, among $E_{1}, E_{2}$, one is stable and the other one unstable.

However, the global stability analysis was not made in [6]. Consider the variables

$$
Z_{1}=S+\frac{1}{Y_{x 1}} X_{1}+\frac{1}{Y_{x 2}} X_{2}, \quad Z_{2}=P-Y_{p 1} X_{1}-Y_{p 2} X_{2}
$$

One can straightforwardly check that $Z_{1}, Z_{2}$ are solutions of the scalar dynamics

$$
\frac{d Z_{1}}{d t}=D\left(S_{\text {in }}-Z_{1}\right), \quad \frac{d Z_{2}}{d t}=-D Z_{2}
$$

from which one deduces that $Z_{1}(t)$ and $Z_{2}(t)$ converge asymptotically to $S_{i n}$ and 0 , respectively. As the solutions are bounded, one can study without loss of generality, the reduced dynamics on the set $Z_{1}=S_{i n}, Z_{2}=0$, that is

$$
\begin{aligned}
& \frac{d X_{1}}{d t}=\mu_{1}\left(S_{i n}-\frac{X_{1}}{Y_{x 1}}-\frac{X_{2}}{Y_{x 2}}, Y_{p 1} X_{1}+Y_{p 2} X_{2}\right) X_{1}-D X_{1} \\
& \frac{d X_{2}}{d t}=\mu_{2}\left(S_{\text {in }}-\frac{X_{1}}{Y_{x 1}}-\frac{X_{2}}{Y_{x 2}}, Y_{p 1} X_{1}+Y_{p 2} X_{2}\right) X_{2}-D X_{2}
\end{aligned}
$$

First, determine the Jacobian matrix at the wash-out equilibrium $E_{0}$

$$
J\left(E_{0}\right)=\left[\begin{array}{cc}
\mu_{1}\left(S_{i n}, 0\right)-D & 0 \\
0 & \mu_{2}\left(S_{i n}, 0\right)-D
\end{array}\right]
$$

Under the condition

$$
D<\min \left\{\mu_{1}\left(S_{i n}, 0\right), \mu_{2}\left(S_{i n}, 0\right)\right\}
$$

$E_{0}$ is a repulsive node and equilibria $E_{1}$ and $E_{2}$ both exist, which corresponds to the most interesting case in view of 
production of $P$. We shall assume that this condition is fulfilled in the following.

On the positive domain $\mathscr{D}=\mathbb{R}_{+} \backslash\{0\} \times \mathbb{R}_{+} \backslash\{0\}$, consider now the variables $\xi_{1}=\ln X_{1}, \xi_{2}=\ln X_{2}$. One has then

$$
\begin{aligned}
& \frac{d \xi_{1}}{d t}=F_{1}(\xi)=\mu_{1}\left(S_{i n}-\frac{e^{\xi_{1}}}{Y_{x 1}}-\frac{e^{\xi_{2}}}{Y_{x 2}}, Y_{p 1} e^{\xi_{1}}+Y_{p 2} e^{\xi_{2}}\right)-D \\
& \frac{d \xi_{2}}{d t}=F_{2}(\xi)=\mu_{2}\left(S_{i n}-\frac{e^{\xi_{1}}}{Y_{x 1}}-\frac{e^{\xi_{2}}}{Y_{x 2}}, Y_{p 1} e^{\xi_{1}}+Y_{p 2} e^{\xi_{2}}\right)-D
\end{aligned}
$$

Then, one obtains

$\operatorname{div} F=e^{\xi_{1}}\left(-\frac{\partial_{S} \mu_{1}}{Y_{x 1}}+Y_{p 1} \partial_{P} \mu_{1}\right)+e^{\xi_{2}}\left(-\frac{\partial_{S} \mu_{2}}{Y_{x 2}}+Y_{p 2} \partial_{P} \mu_{2}\right)$

which is negative on $\mathscr{D}$. From Dulac criterion, we deduce that the dynamics does not have periodic solution.

Notice that when the equilibria $E_{1}, E_{2}$ are unstable, their stable manifold belongs to the axis $x_{1}$ or $x_{2}$. Notice also that the Jacobian matrix of the system (7)-(8) at a coexistence equilibrium $E_{\star}$ is

$$
J\left(E_{\star}\right)=\left[\begin{array}{cc}
\left(-\frac{\partial_{S} \mu_{1}}{Y_{x 1}}+Y_{p 1} \partial_{P} \mu_{1}\right) X_{1} & \left(-\frac{\partial_{S} \mu_{1}}{Y_{x 2}}+Y_{p 2} \partial_{P} \mu_{2}\right) X_{1} \\
\left(-\frac{\partial_{S} \mu_{1}}{Y_{x 1}}+Y_{p 1} \partial_{P} \mu_{1}\right) X_{2} & \left(-\frac{\partial_{S} \mu_{1}}{Y_{x 2}}+Y_{p 2} \partial_{P} \mu_{2}\right) X_{2}
\end{array}\right]
$$

and its trace is negative. Hence, when $E_{\star}$ exists and is unstable, it is necessarily a saddle point and as it is the only unstable positive equilibrium and there exist exactly two stable equilibria (either $E_{1}$ and $E_{2}$ if $E_{\star}$ is the only equilibrium, or the other positive equilibrium and one among $E_{1}, E_{2}$ ), its stable manifold is the separatrix in the $\left(X_{1}, X_{2}\right)$ plane between the attraction basins of the two stable equilibria. Therefore, in any case, the system does not have any polycycle. Finally, we conclude by Poincaré-Bendixon Theorem, that any solution converges to an equilibrium point.

Let us now analysis the situation with respect to the productivity, which is defined, at steady state, by the quantity $\pi:=D P$. From the expressions (5) and the implicit function theorem, one obtain that the the curves $\mathscr{C}_{i}$ can be described as the graphs of functions $S \mapsto P_{i}(S)$ in the $(S, P)$ plane with

$$
P_{i}^{\prime}=-\frac{\partial_{S} \mu_{i}}{\partial_{P} \mu_{i}}>0
$$

Therefore the functions $P_{i}(\cdot)$ are increasing. We deduce that any intersection of $\mathscr{C}_{1}$ with $\mathscr{C}_{2}$ that lies between $\mathscr{L}_{1}$ and $\mathscr{L}_{2}$ has necessarily a value of $P$ between the ones of the intersections $\mathscr{L}_{1} \cap \mathscr{C}_{1}$ and $\mathscr{L}_{2} \cap \mathscr{C}_{2}$. Furthermore, the intersection $\mathscr{L}_{i} \cap \mathscr{C}_{i}(i=1,2)$ that possesses the largest value of $P$ is the one with the largest number $Y_{x i} Y_{p i}$. This amounts to say that any coexistence equilibrium $E_{\star}$, if it exists, has a value of $P$ smaller than the equilibrium $E_{i}$ with the largest number $Y_{x i} Y_{p i}$. Consequently, the best productivity is necessarily reached with this species $i$ alone. The productivity associated to the equilibrium $E_{i}$ can be written as a function of $S$ :

$$
\begin{aligned}
\pi_{i}(S) & =\mu_{i}\left(S, Y_{x i} Y_{p i}\left(S_{i n}-S\right)\right) Y_{x i} Y_{p i}\left(S_{i n}-S\right) \\
& =\frac{\mu_{m i} K_{p i} Y_{x i} Y_{p i} S}{\left(K_{s i}+S\right)\left(K_{p i}+Y_{x i} Y_{p i}\left(S_{i n}-S\right)\right)}\left(S_{i n}-S\right)
\end{aligned}
$$

This function is well defined on the interval $\left[0, S_{i n}\right]$ with $\pi_{i}(0)=0, \pi\left(S_{i n}\right)=0$ and is positive on $\left(0, S_{i n}\right)$. Therefore it admits a maximum. A straightforward calculation gives

$$
\pi^{\prime}(S)=-\mu_{m i} K_{p i} Y_{x i} Y_{p i} \frac{A_{i} S^{2}+2 B_{i} S-C_{i}}{\left(K_{s i}+S\right)^{2}\left(K_{p i}+Y_{x i} Y_{p i}\left(S_{i n}-S\right)\right)^{2}}
$$

with $A_{i}=K_{p i}-K_{s i} Y_{x i} Y_{p i}, B_{i}=K_{s i}\left(K_{p i}+S_{i n} Y_{x i} Y_{p i}\right)$ and $C_{i}=$ $K_{s i} S_{i n}\left(K_{p i}+S_{i n} Y_{x i} Y_{p i}\right)$. Then, the maximum of $\pi_{i}$ on $\left(0, S_{i n}\right)$ is unique and reached for

$$
S_{i}^{\dagger}=\frac{-B_{i}+\operatorname{sign}\left(A_{i}\right) \sqrt{\Delta_{i}}}{A_{i}}
$$

where $\Delta_{i}=K_{p i} K_{s i}\left(K_{s i}+S_{i n}\right)\left(S_{i n} Y_{x i} Y_{p i}+K_{p i}\right)>0$, and the corresponding value of the dilution rate is

$$
D_{i}^{\dagger}=\mu_{i}\left(S_{i}^{\dagger}, Y_{x i} Y_{p i}\left(S_{i n}-S_{i}^{\dagger}\right)\right)
$$

However, this equilibrium $E_{i}^{\dagger}$ is not stable when $\mu_{j}\left(S_{i}^{\dagger}, Y_{x i} Y_{p i}\left(S_{i n}-S_{i}^{\dagger}\right)\right)>D_{i}^{\dagger}$, where $j$ denotes the label of the other species. If it is stable, there exists another stable equilibrium (either $E_{j}$ or a coexistence equilibrium $E_{\star}$ ). In both cases, a feedback law is required to stabilize or enlarge its attraction basin. This is the matter of the next section.

\section{A GAIN-SCHEDULING APPROACH}

As shown in the previous section, the productivity under constant control depends on which species wins the competition or whether or not the coexistence is possible. Therefore, the control goal is to stabilize the product concentration (thus, the productivity) at the desired level $P_{s p}$. Since the considered system is highly nonlinear, we propose a controller that is based on output feedback linearization [7]. The idea is to find a control law so that the equation governing the concentration of product $P$ in closed-loop is linear exponentially stable. If the process variable is the product concentration $P$ and the manipulated variable $u$ is the dilution rate $D$, then is clearly seen that the relative degree of system (1)-(4) is equal to $r=1$ for $P>0$, and from (4) we have

$$
\frac{d P}{d t}=-u P+\mu_{1}(S, P) Y_{p 1} X_{1}+\mu_{2}(S, P) Y_{p 2} X_{2}
$$

Assuming that the specific growth rates are known and that the state variables are measured at every time instant $t$, the manipulated variable $u=D$ is chosen as follows

$$
u=\frac{\mu_{1}(S, P) Y_{p 1} X_{1}+\mu_{2}(S, P) Y_{p 2} X_{2}-k\left(P_{s p}-P\right)}{P}
$$

Then, by substituting (10) into (4) we have

$$
\frac{d P}{d t}=k\left(P_{s p}-P\right)
$$

where $k>0$ is the only controller parameter that determines the convergence rate of $P$ to the set point product concentration $P_{s p}$. Let us underline that the proposed controller can be easily implemented for a biological wastewater treatment plant, where, as shown in [16], the precise knowledge of the functions $\mu_{i}$ is not required when $P$ and the output gas flow rate are the only measurements (as this later one is proportional to $\left.\mu_{1}(S, P) Y_{p 1} X_{1}+\mu_{2}(S, P) Y_{p 2} X_{2}\right)$. 
When implementing the control law (10), we must take into account that the manipulated variable $D$ has to take values within a prescribed interval $\left[D_{\min }, D_{\max }\right]$, while the expression of $u$ may give values outside this physical interval. Indeed, if $P_{s p}>P$ and $k$ is sufficiently large, then the expression of $u$ is negative. If the numerator in (10) is positive, but the product concentration $P$ is close to zero, then $u$ may take very large values. In practice, one uses a saturated expression of $u$.

Moreover, the control law (10) does not ensure the global stability of the closed-loop system, but it is possible to find the largest positively invariant set that includes the desired product concentration $P_{s p}$.

As shown previously with (6), $S=S_{i n}-X_{1} / Y_{x 1}-X_{2} / Y_{x 2}$ and $P=X_{1} Y_{p 1}+X_{2} Y_{p 2}$ define a global attractor for the system (1)-(4). Hence, the manipulated variable (10) can be expressed as a function of $X_{1}$ and $X_{2}$ for the reduced dynamics in the plane $\left(X_{1}, X_{2}\right)$ and it is possible to determine the borders $u\left(X_{1}, X_{2}\right)=D_{\min }$ and $u\left(X_{1}, X_{2}\right)=D_{\max }$ corresponding to lower and upper bounds of the manipulated variable (10).

The closed-loop system (7)-(8), (10) with $P_{s p}>0$ has the following equilibrium points: $E_{1}^{c}$ with a single species $X_{1}$ and $P=P_{s p}=Y_{p 1} X_{1}, E_{2}^{c}$ with a single species $X_{2}$ and $P=P_{s p}=$ $Y_{p 2} X_{2}$, and $E_{\star}^{c}$ with two coexisting species and $P=P_{s p}=$ $Y_{p 1} X_{1}+Y_{p 2} X_{2}$. Please note that in the closed-loop system, there is at most one equilibrium point corresponding to the coexistence case. Let $R:=\mu_{1}-\mu_{2}$, then at the equilibrium point $E_{\star}^{c}$ we have

$$
R=\frac{\mu_{m 1} S}{S+K_{s 1}} \cdot \frac{K_{p 1}}{K_{p 1}+P_{s p}}-\frac{\mu_{m 2} S}{S+K_{s 2}} \cdot \frac{K_{p 2}}{K_{p 2}+P_{s p}}=0
$$

and (12) has at most one solution $S \in\left(0, S_{i n}\right)$. The corresponding species concentrations at $E_{\star}^{c}$ are found from (1) and (4).

The Jacobian matrix determined at $E_{1}^{c}$ is

$$
J\left(E_{1}^{c}\right)=\left[\begin{array}{cc}
\frac{\partial}{\partial X_{1}}\left(\mu_{1}-D\right) \cdot X_{1} & \frac{\partial}{\partial X_{2}}\left(\mu_{1}-D\right) \cdot X_{1} \\
0 & \mu_{2}-D
\end{array}\right]
$$

and the closed-loop system is locally asymptotically stable at $E_{1}^{c}$, when

$$
\left.\frac{\partial}{\partial X_{1}}\left(\mu_{1}-D\right)\right|_{E_{1}^{c}}=\frac{-k Y_{p 1}}{P_{s p}}<0, \quad \mu_{2}-D=-\left.R\right|_{E_{1}^{c}}<0
$$

If $R<0$ at $E_{1}^{c}$, then $E_{1}^{c}$ is a saddle-point. Similarly, the Jacobian matrix determined at $E_{2}^{c}$ is

$$
J\left(E_{2}^{c}\right)=\left[\begin{array}{cc}
\mu_{1}-D & 0 \\
\frac{\partial}{\partial X_{1}}\left(\mu_{2}-D\right) \cdot X_{2} & \frac{\partial}{\partial X_{2}}\left(\mu_{2}-D\right) \cdot X_{2}
\end{array}\right]
$$

and the closed-loop system is locally asymptotically stable at $E_{2}^{c}$, when

$$
\left.\frac{\partial}{\partial X_{2}}\left(\mu_{2}-D\right)\right|_{E_{2}^{c}}=\frac{-k Y_{p 2}}{P_{s p}}<0, \quad \mu_{1}-D=\left.R\right|_{E_{2}^{c}}<0
$$

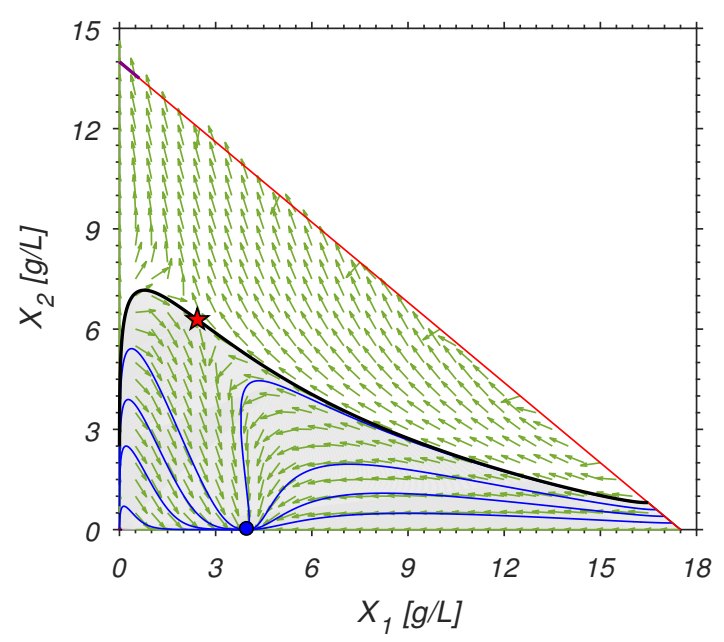

Fig. 2. Trajectories in the closed-loop system with unstable coexistence point $E_{\star}^{c}$ (red star) for $P_{s p}=8.0[\mathrm{~g} / \mathrm{L}]$ and $k=0.001[1 / \mathrm{h}]$

If $R>0$ at $E_{2}^{c}$, then $E_{2}^{c}$ is a saddle-point. Finally, at the coexistence equilibrium point $E_{\star}^{c}$, the Jacobian matrix is as follows

$$
J\left(E_{\star}^{c}\right)=\left[\begin{array}{cc}
\frac{\partial}{\partial X_{1}}\left(\mu_{1}-D\right) \cdot X_{1} & \frac{\partial}{\partial X_{2}}\left(\mu_{1}-D\right) \cdot X_{1} \\
\frac{\partial}{\partial X_{1}}\left(\mu_{2}-D\right) \cdot X_{2} & \frac{\partial}{\partial X_{2}}\left(\mu_{2}-D\right) \cdot X_{2}
\end{array}\right]
$$

and the corresponding characteristic equation is

$$
(\lambda+k)(\lambda+W)=0
$$

where $W=\left(\partial_{X_{2}} R \cdot Y_{p 1}-\partial_{X_{1}} R \cdot Y_{p 2}\right) X_{1} X_{2} / P_{s p}$. Then, the closed-loop system is locally asymptotically stable at $E_{\star}^{c}$ if $W>0$, unstable if $W<0$ and $E_{\star}^{c}$ is a saddle-point.

If all the equilibrium points exist and the closed-loop system (7)-(8) with (10) is asymptotically stable at $E_{\star}^{c}$, then $E_{1}^{c}$ and $E_{2}^{c}$ are saddle-points. When the closed-loop system is unstable at $E_{\star}^{c}$, then it is locally asymptotically stable at $E_{1}^{c}$ and $E_{2}^{c}$, and the control goal $P_{s p}$ is obtained. As shown in Section II, the largest value of product concentration is obtained for a single species $X_{i}$ with the largest number $Y_{x i} Y_{p i}$ $(i=1,2)$. Fig. 2 shows the case, where the desired product concentration $P_{s p}$ can only be obtained for a single species $X_{1}$ and the closed-loop system is unstable at the coexistence point $E_{\star}^{c}$. The red line defines a subset in $\left(X_{1}, X_{2}\right)$ with nonnegative substrate concentrations. Since $E_{\star}^{c}$ is the saddlepoint (red star), there exist a stable manifold (separatrix trajectories - black curves) that allow us to define the largest invariant subset including the equilibrium point $E_{1}^{c}$ (blue point) with the desired product concentration $P_{s p}$. If the trajectories (blue curves) start inside the invariant set, the control goal is achieved. For a given set point product concentration $P_{s p}$, the size of the invariant set depends on the controller parameter $k$. Moreover, according to (11) the convergence rate of $P$ to $P_{s p}$ is strictly determined by $k$. Figs. 3 and 4 show the invariant sets for two different values of the parameter $k$. To achieve the desired product concentration $P_{s p}$ for a larger basin of attraction, we propose 


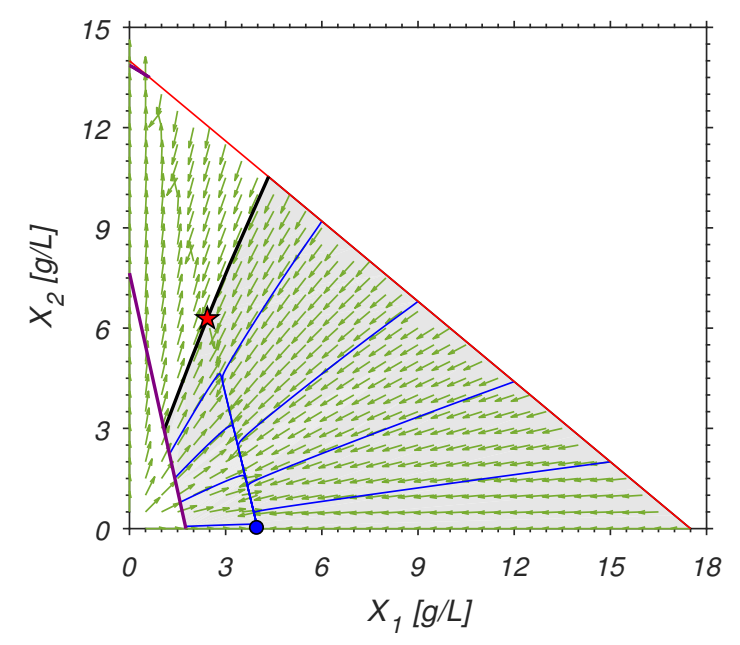

Fig. 3. Trajectories in the closed-loop system with unstable coexistence point $E_{\star}^{c}$ (red star) for $P_{s p}=8.0[\mathrm{~g} / \mathrm{L}]$ and $k=0.1[1 / \mathrm{h}]$

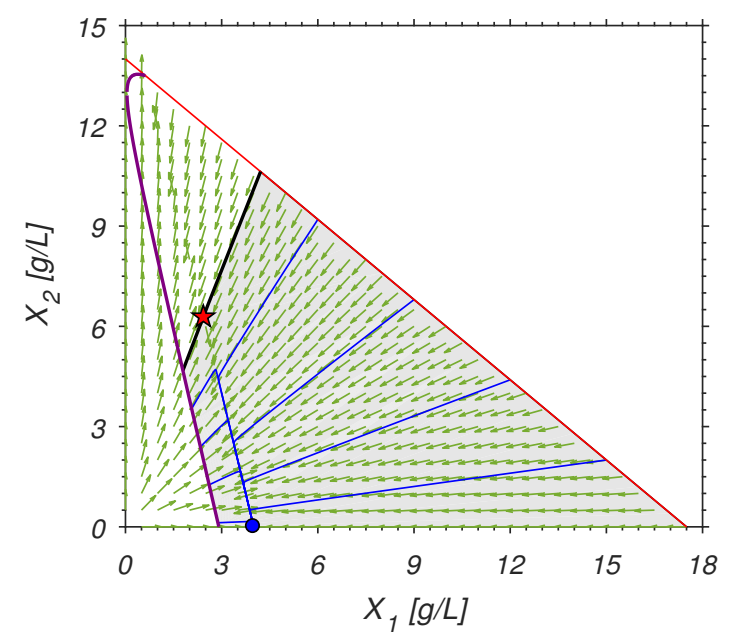

Fig. 4. Trajectories in the closed-loop system with unstable coexistence point $E_{\star}^{c}$ (red star) for $P_{s p}=8.0[\mathrm{~g} / \mathrm{L}]$ and $k=0.25[1 / \mathrm{h}]$

to design a family of feedback linearizing controllers for different values of $k$. Then, if the trajectory enters the region with a larger $k$, the controller parameter $k$ is switched to a new value. Since the invariant regions can overlap, the largest possible value of $k$ is always chosen. Hence, the proposed idea is similar to the well-known gain-scheduling approach. When constructing the invariant regions for different values of $k$, constraints for the manipulated variable can be easily included. The violet lines in Figs. 3 and 4 represent the subsets in the $\left(X_{1}, X_{2}\right)$ plane where $u=0$, which are the boundaries of the regions for which $u$ is negative. The effectiveness of the proposed control algorithm is presented in the next section.

\section{NUMERICAL ILlUStRATIONS}

The simulations are obtained for (7)-(8) with (10) and the following parameters: $\mu_{m 1}=0.3[1 / \mathrm{h}], \mu_{m 2}=0.25[1 / \mathrm{h}], K_{s 1}=$

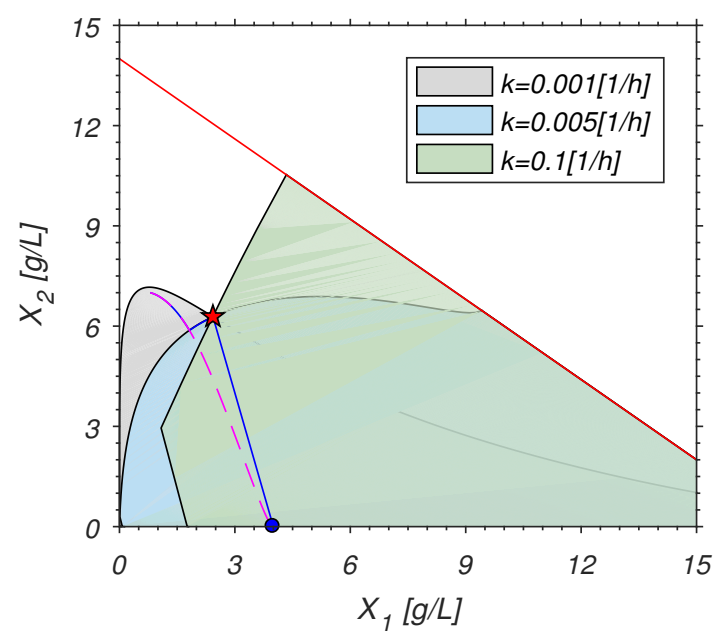

Fig. 5. Trajectories in the closed-loop system with (blue line) and without gain-scheduling (dashed magenta line) approach

$2.7[\mathrm{~g} / \mathrm{L}], K_{s 2}=1.5[\mathrm{~g} / \mathrm{L}], K_{p 1}=3.0[\mathrm{~g} / \mathrm{L}], K_{p 2}=3.5[\mathrm{~g} / \mathrm{L}]$, $Y_{x 1}=0.5[\mathrm{~g} / \mathrm{g}], Y_{x 2}=0.4[\mathrm{~g} / \mathrm{g}], Y_{p 1}=2.0[\mathrm{~g} / \mathrm{g}], Y_{p 2}=0.5[\mathrm{~g} / \mathrm{g}]$, $S_{\text {in }}=35.0[\mathrm{~g} / \mathrm{L}]$, and $P_{s p}=8.0[\mathrm{~g} / \mathrm{L}]$. In this case, the desired product concentration $P_{s p}$ can only be achieved for a single species $X_{1}$ corresponding to equilibrium point $E_{1}^{c}$. For $P_{s p}=8.0[\mathrm{~g} / \mathrm{L}]$ the equilibrium point $E_{2}^{c}$ does not exist and the closed-loop system at the coexistence equilibrium $E_{\star}^{c}$ is unstable. Hence, a family of three feedback linearizing controllers is designed for $k \in\{0.001,0.005,0.1\}[1 / \mathrm{h}]$ and the lower and upper bounds for the manipulated variable are equal to $D_{\min }=0.001[1 / \mathrm{h}]$ and $D_{\max }=0.3[1 / \mathrm{h}]$, respectively. Fig. 5 shows trajectories in the closed-loop system with constant $k=0.001[1 / \mathrm{h}]$ and for the proposed controller, where the parameter $k$ is switched depending on which invariant region is active. It is clearly seen that the basin of attraction can be significantly enlarged, if the parameter $k$ is modified. Fig. 6 is a close-up view of the trajectories near a saddle equilibrium point $E_{\star}^{c}$. The corresponding time courses of the product concentration $P(t)$ and the manipulated variable (dilution rate $D(t)$ ) are shown in Figs. 7 and 8. Starting from the same initial conditions in $\left(X_{1}, X_{2}\right)$, the convergence rate to the desired product concentration $P_{s p}$ is significantly increased for the proposed controller in comparison to the feedback linearizing control with a constant parameter $k=$ $0.001[1 / \mathrm{h}]$. Moreover, in each case the manipulated variable is never saturated.

\section{CONCLUSION}

In the present paper we have proposed a family of feedback linearizing controllers for a chemostat system with two competing species for a single growth limiting substrate, but in the presence of an internal inhibitor. The inhibitor was a product $P$ secreted by both microbial species $X_{1}$ and $X_{2}$. The control goal was to achieve the desired productivity by controlling the product concentration $P$ at a desired level $P_{s p}$. First, depending on the dilution rate $D$, we have shown that the open-loop system can have up to five equilibrium 


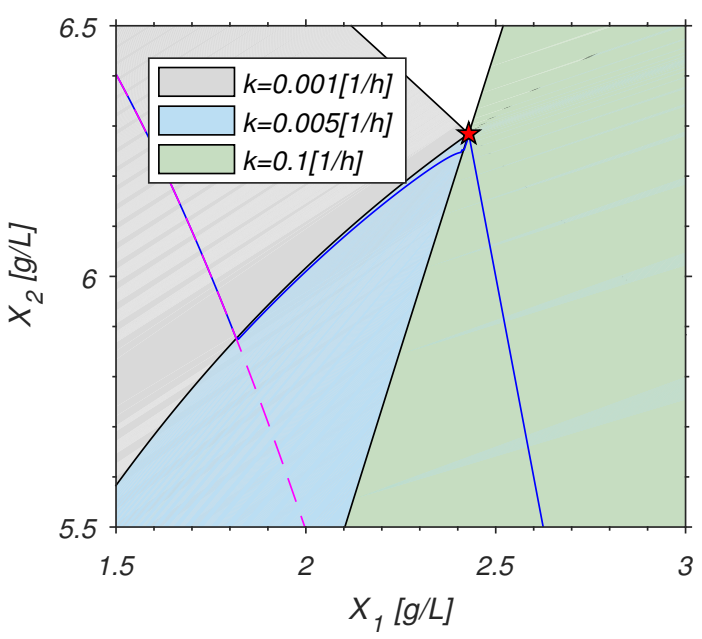

Fig. 6. Trajectories in the closed-loop system near unstable equilibrium point $E_{\star}^{c}$ (red star)

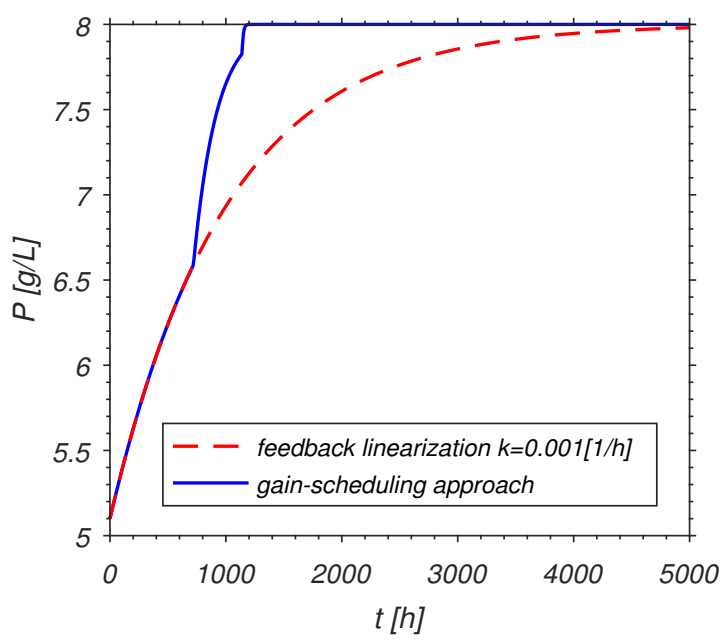

Fig. 7. Time courses of product concentration $P(t)=Y_{p 1} X_{1}(t)+Y_{p 2} X_{2}(t)$ in the closed-loop system with and without gain-scheduling approach

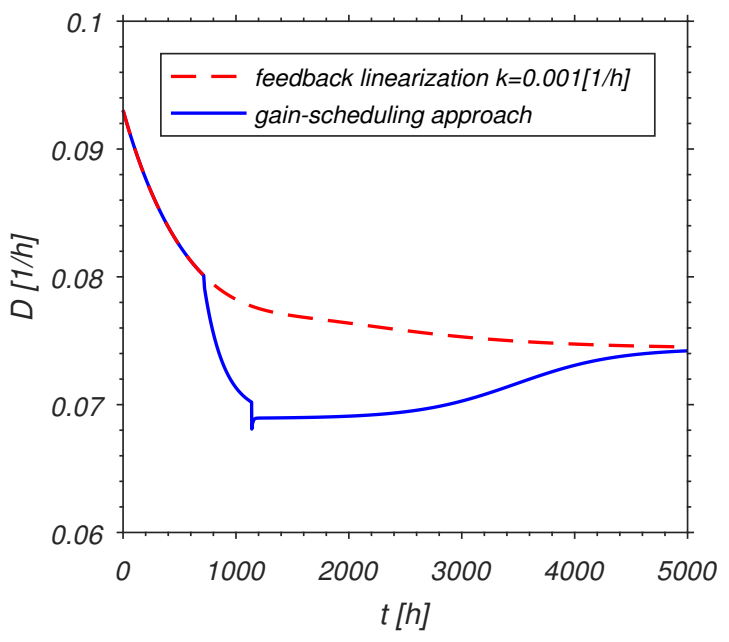

Fig. 8. Time courses of manipulated variable $D(t)$ in the closed-loop system with and without gain-scheduling approach points (including two coexistence equilibria) and we have derived conditions for the global stability at the coexistence equilibrium point. Moreover, we have shown that the best productivity is always obtained with a single species. Then, we have analyzed the possible equilibrium points in the closed-loop system, with an output feedback linearizing controller. Finally, we showed that for the proposed controller, the domain of attraction can be enlarged and the set point product concentration $P_{s p}$ can be quickly achieved. We believe that the gain-scheduling approach that we propose here could be applied in more general cases, which could be the matter of a future work.

\section{ACKNOWLEDGMENTS}

This work was partially supported by the Polish Ministry of Science and Higher Education under grant 02/010/BKM18/0136 (PS) and grant of Silesian University of Technology 02/010/RGJ18/0107 (PS).

\section{REFERENCES}

[1] H. Smith and P. Waltman, The Theory of Chemostat - Dynamics of microbial competition, Cambridge University Press - Cambridge Studies in Mathematical Biology, 1995.

[2] J. Harmand, C. Lobry, A. Rapaport and T. Sari, The Chemostat: mathematical theory of microorganisms cultures, ISTE Wiley, 2017.

[3] S.B. Hsu and P. Waltman, A survey of mathematical models of competition with an inhibitor, Mathematical biosciences 187, 53-91, 2004

[4] M. Dellal, M. Lakrib and T. Sari, The operating diagram of a model of two competitors in a chemostat with an external inhibitor, Mathematical biosciences 302, 27-45, 2018.

[5] S. Roy, The coevolution of two phytoplankton species on a single resource: allelopathy as a pseudo-mixotrophy, Theoretical Population Biology 75, 68-75, 2009.

[6] M. De Freitas and A. Fredrickson, Inhibition as a Factor in the Maintenance of the Diversity of Microbial Ecosystems, Microbiology 106, 307-320, 1978

[7] M.A. Henson and D.E. Seborg, Nonlinear control strategies for continuous fermenters, Chemical Engineering Science, 47, 821-835, 1992.

[8] G.P. Reddy and M. Chidambaram, Near-optimal productivity control of a continuous bioreactor, IEE Proceedings-Control Theory and Applications, 142, 633-637, 1995.

[9] J.Y. Dieulot, A productivity signal feedback controller for continuous bioreactors, Journal of Process Control, 22, 1318-1324, 2012.

[10] S. Ramaswamy, T.J. Cutright, and H.K. Qammar, Control of a continuous bioreactor using model predictive control, Process Biochemistry, 40, 2763-2770, 2005

[11] A. Ashoori, B. Moshiri, A. Khaki-Sedigh, and M.R. Bakhtiari, Optimal control of a nonlinear fed-batch fermentation process using model predictive approach, Journal of Process Control, 19, 1162-1173, 2009.

[12] R. Aguilar, J. Gonzalez, M. Barron, R. Martinez-Guerra, and R. MayaYescas, Robust $\mathrm{PI}^{2}$ controller for continuous bioreactors, Process Biochemistry, 36, 1007-1013, 2001.

[13] G. Szederkényi, N.R. Kristensen, K.M. Hangos, and S.B. Jørgensen, Nonlinear analysis and control of a continuous fermentation process, Computers and Chemical Engineering, 26, 659-670, 2002.

[14] H. De Battista, M. Jamilis, F. Garelli, and J. Picó, Global stabilisation of continuous bioreactors: Tools for analysis and design of feeding laws, Automatica, 89, 340-348, 2018.

[15] H. Noussi, M. Ballyk, and E. Barany, Stabilization of chemostats using feedback linearization, In 2007 46th IEEE Conference on Decision and Control, 677682, IEEE, 2007.

[16] R. Antonelli, J. Harmand, J.P. Steyer, and A. Astolfi, Set-point regulation of an anaerobic digestion process with bounded output feedback, IEEE Transactions on control systems technology, 11, 495-504, 2003.

[17] A. Schaum, J. Alvarez, and T. Lopez-Arenas, Saturated PI control of continuous bioreactors with Haldane kinetics, Chemical engineering science, 68, 520-529, 2012. 